\title{
A high level of satisfaction after bicompartmental individualized knee arthroplasty with patient-specific implants and instruments
}

\author{
Takahiro Ogura ${ }^{1} \cdot$ Kiet Le $^{2} \cdot$ Gergo Merkely ${ }^{3} \cdot$ Tim Bryant $^{4} \cdot$ Tom Minas $^{4}$
}

Received: 16 March 2018 / Accepted: 19 September 2018 / Published online: 5 October 2018

(c) The Author(s) 2018

\begin{abstract}
Purpose Customized Individually Made (CIM) Bicompartmental Knee Arthroplasty (BKA) implants and three-dimensional printed customized instruments are available to fit to each patient's unique anatomy, medial or lateral with patellofemoral. This study aimed to evaluate the clinical outcomes after CIM-BKA.

Methods Fifty-five patients [59 knees; average age, 51 years; standard deviation (SD), 6.8; range 37-65 years] who underwent CIM-BKA were evaluated over an average of 3.8-year follow-up (SD 1.6; range 1-6 years). Forty-one knees underwent BKA combined medial and patellofemoral replacement (BKA-MP) and 18 knees underwent BKA combined lateral with patellofemoral replacement (BKA-LP). Survival rates, the modified Cincinnati Knee Rating Scale, WOMAC, VAS, SF-36, a satisfaction survey, and radiographic evaluation were used to evaluate outcomes.

Results Overall, survival rates were $98 \%$ and $92 \%$ at 2 and 5 years, respectively. Of 56 knees (95\%) that did not fail, all patient-reported functional scores significantly improved post-operatively $(P<0.01)$, regardless of the previous surgeries, with a high level of satisfaction (51/56 knees, $91 \%)$. Radiographically, all the femoral components fit perfectly and 56 knees (95\%) of the tibial components fit with less than $2 \mathrm{~mm}$ of undercoverage or overhang. Three knees (5\%) required the conversion to TKA and 17 knees (29\%) required subsequent surgical procedures, of which multiply operated knees had higher rate than virgin knee [14/40 (35\%) vs. 3/16 (19\%)].

Conclusion CIM-BKA allowed precise fit of the components and provided a significant improvement post-operatively with a high level of satisfaction over short- to mid-term follow-up. This novel CIM-BKA is resurfacing, and does not require 10-mm faceted cuts, being only 3-mm-thick, which preserves bone stock for the future. It may be a promising option for relatively young active patients with bicompartmental osteoarthritis with a longer term follow-up being necessary.
\end{abstract}

Level of evidence IV.

Keywords Bicompartmental knee arthroplasty; osteoarthritis · Resurfacing $\cdot$ Customized individually made $\cdot$ Patientspecific implantation

\section{Abbreviations}

OA Osteoarthritis

BKA Bicompartmental knee arthroplasty

Tom Minas

tminas@paleyinstitute.org

1 Sports Medicine Center, Funabashi Orthopaedic Hospital, 1-833 Hasama, Funabashi, Chiba 274-0822, Japan

2 Newton-Wellesley Sports Medicine, 2000 Washington St., Blue Building, Suite 423, Newton, MA 02462, USA

3 Department of Traumatology, Semmelweis University, Uzsoki u. 29, Budapest 1145, Hungary

4 Cartilage Repair Center, Paley Orthopedic and Spine Institute, St. Mary's Hospital, 901 45th Street, Kimmel Building, West Palm Beach, FL 33407, USA
CIM Customized individually made

TKA Total knee arthroplasty

\section{Introduction}

Osteoarthritis (OA) of the knee is one of the most common diseases that cause pain and dysfunction [17] and affects many health outcomes [16]. For the treatment of OA, total knee arthroplasty (TKA) is the standard of care that provides high reproducibility of outcomes except in young patients where the failure rate is higher, and patient satisfaction and improvement are lower than older patients $[10,18,26,29]$. Recently, a systematic review showed that, in patients under 65 years old, unicompartmental 
knee arthroplasty (UKA) provided higher functional outcomes but higher revision rates than TKA [14]. Therefore, treating $\mathrm{OA}$ in young patients is challenging.

For those who have localized arthritis rather than tricompartmental arthritis, however, sacrificing the other unaffected compartment and cruciate ligaments by performing TKA is concerning, as cruciates play an important role on load distribution, stability [30], and possibly proprioception [11]. Preservation of bone stock is desirable when considering future revision surgeries in a young active patient. A pattern of wear with bicompartmental OA is commonly observed in patients who undergo TKA. To manufacture a bicompartmental arthroplasty, a computer tomography (CT)-based, customized, patient-specific Bicompartmental Knee Arthroplasty (BKA) and printed nylon individualized instruments have been developed. Customized Individually Made (CIM) BKA implants (ConforMIS Inc, Burlington, MA) and three-dimensional printed customized surgical instruments are currently available to fit to each patient's unique anatomy, medial and patellofemoral, or lateral and patellofemoral. Unique to this implant is that it resurfaces the $3 \mathrm{~mm}$ of cartilage on the subchondral bone with a 3-mm-thick implant and only removes a 3-5-mm anterior and posterior femoral cut to implant the prosthesis. Historically, a bicompartmental monolithic implant, made from a standard prosthesis less a condyle using standardized faceted $10 \mathrm{~mm}$ cuts, has been available in the past but taken off the market because of poor outcomes due to impingement and fit issues. These occurred at the patellofemoral native compartment junction as well as tibial baseplate fractures resulting in a high failure rate of up to $60 \%$ at a minimum follow-up of 54 months $[8,22]$. Otherwise, modular "off-the-shelf" implants using unicondylar implants combined with a patellofemoral implant are required. However, controversy still exists regarding the functional outcomes when compared to TKA [23, 31]. To date, scarce data are available on the treatment of bicompartmental osteoarthritis. The outcomes of this novel resurfacing CIM-BKA have not been previously reported. The aim of this study was to evaluate the clinical outcomes and patient satisfaction of patients after CIM-BKA, previously unreported, over the short- to mid-term follow-up. The hypothesis in this study was that a significant improvement in pain and function with a high level of satisfaction would be provided after CIM-BKA while preserving the cruciates and bone stock.

\section{Materials and methods}

A total of 55 patients underwent CIM-BKA (ConforMIS Inc) for symptomatic bicompartmental OA (combined medial or lateral with patellofemoral OA) between September 2010 and February 2016. A single surgeon performed all procedures. This cohort represented approximately $10 \%$ of the cases performed during this period for which TKAs were performed, average 100/year. Of this cohort, we evaluated all 55 patients (59 knees; average age, 51 years; SD 6.8; range 37-65) over an average of 3.8-year follow-up [standard deviation (SD) 1.6; range 1-6 years] as all had successfully completed more than 1 year of follow-up by the time of data analysis. There were 35 women and 24 men with an average body mass index (BMI) of 28.3 (SD 4.8; range 19.2-39.3 kg/m²). A total of 41 knees underwent BKA combined medial and patellofemoral replacement (BKA-MP), and 18 knees underwent BKA combined lateral and patellofemoral replacement (BKA-LP) (Table 1). Simultaneous bilateral procedure was performed in 1 patient. Indications for this surgery were bicompartmental joint disease including combined medial or lateral with patellofemoral OA. Contraindications to this surgery included tricompartmental OA, ligament instabilities, or knee deformities greater than $15^{\circ}$. Patients who had prior high tibial osteotomy (HTO) were not considered contraindication. Standing longalignment radiographs to include hip/knee/ankle, standing anteroposterior (AP), Rosenberg, lateral radiographs, and skyline view were evaluated pre-operatively. In addition, either MRI or CT arthrography was performed pre-operatively to assess the whole joint before considering BKA.

Prior to the index surgery, 42 of all 59 knees $(71 \%)$ had a total of 141 procedures (mean 3.4; SD 2.8; range 1-13)

Table 1 Patient demographics

BKA-MP $(n=41$ knees $)$

BKA-LP $(n=18$ knees $)$

Overall ( $n=59$ knees $)$

Variables

Age at surgery (years), mean \pm SD (range)

Gender, male/female, $n$

Right/left knee, $n$

Body mass index $\left(\mathrm{kg} / \mathrm{m}^{2}\right)$, mean $\pm \mathrm{SD}$ (range)

$48.6 \pm 6.4(37-60)$
$8 / 10$
$10 / 8$
$27.1 \pm 6.4(19.2-39.3)$

$50.7 \pm 6.8(37-65)$

$24 / 35$

$32 / 27$

$28.2 \pm 4.8(19.2-39.3)$

$B K A-M P$ bicompartmental knee arthroplasty combined medial and patellofemoral replacement, $B K A-L P$ bicompartmental knee arthroplasty combined lateral with patellofemoral replacement, $S D$ standard deviation 
primarily including a total of 107 arthroscopic procedures (primarily partial meniscectomy and chondroplasty) in 39 knees (mean 2.7; SD 2.2; range 1-11) (Table 2). Pre-operative AP and lateral radiographs were scored in accordance with the $\mathrm{K}-\mathrm{L}$ grade [13] to evaluate the progression of OA before the index surgery. Radiographic evaluation showed that $61 \%$ knees were grade 2, 29\% were grade 3, and $10 \%$ were grade 4 based on the Kellgren-Lawrence $(\mathrm{K}-\mathrm{L})$ grading system.

\section{Operative technique}

Based on the CT imaging of the affected lower extremity (from hip to ankle), the patient-specific instruments and implants are manufactured. The surgical techniques have been described elsewhere in detail [20, 27]. Briefly, a medial or lateral parapatellar arthrotomy is performed dependent on the compartments treated. The patella is resurfaced in the standard fashion for TKA with an off-the-shelf polyethylene button. The remaining cartilage in the affected trochlea and femoral condylar compartments is removed using ring curettes and a \#10-blade. Then, the osteophytes are removed to the native cortex (confirmed with the pre-operatively

Table 2 Number of knees with the previous surgical procedures

\begin{tabular}{lc}
\hline Procedure & $\begin{array}{l}\text { Number } \\
\text { of knees }\end{array}$ \\
\hline A/S chondroplasty and meniscectomy & 39 \\
ACI total & 8 \\
ACI combined with osteotomy ${ }^{\mathrm{a}}$ & 5 \\
ACI alone & 2 \\
ACI combined with MAT & 1 \\
Osteotomy alone total & 8 \\
HTO & 5 \\
TTO & 2 \\
Extension femoral osteotomy & 1 \\
ACLR & 4 \\
Osteochondral allograft transplantation & 2 \\
OATS combined with TTO & 1 \\
Unicompartmental knee replacement & 1 \\
Others & 4 \\
No previous procedures & 17 \\
\hline
\end{tabular}

The 141 previous procedures were performed among 42 knees (71\%). Some patients had more than one procedure

Others included removal of hardware in 1 and drainage of wound infection in 1

$A / S$ arthroscopic, TTO tibial tubercle osteotomy, HTO high tibial osteotomy, $A C I$ autologous chondrocyte implantation, MAT meniscal allograft transplantation, $A C L R$ anterior cruciate ligament reconstruction, OATS osteochondral autologous transplantation

${ }^{a}$ Osteotomy included tibial tubercle osteotomy [TTO] in 2, HTO in 2, and TTO/distal femoral osteotomy [DFO] in 1 planned ' $\mathrm{iView}{ }^{\mathrm{TM}}$ ' protocol.) The knee joint is balanced in extension and flexion using the patient-specific "balancing chip" jigs, with both anterior and posterior cruciate ligaments being preserved. Tibial bone cuts are performed with the balancing chip jigs being linked to the tibial cutting guide. No distal bone was removed from the femur as the implants fits directly on the subchondral bone plate. Thin anterior and posterior cuts of the anterior trochlea and posterior femoral condyle allow the placement of the trial nylon printed implant. The femoral subchondral bone is prepared for cement penetration with multiple 2-mm drills holes with a step drill to improve cement inter-digitation. The final cobalt-chrome patient-specific femoral implant is cemented in place after the tibial component is cemented with a 5 - or 7-mm polyethylene trial insert. The final polyethylene tibial component is inserted into the tibial tray (Fig. 1). The wound is closed in layers with a soft-compressive dressing, over a drain that is removed the next day.

\section{Post-operative course}

Post-operatively, patients were allowed full weight-bearing and range of motion on the next day of the surgery. The standard thrombosis prophylaxis using $325 \mathrm{mg}$ ASA bid for 3 weeks was utilized. Functional rehabilitation was performed in the same manner as a conventional TKA.

\section{Survival analysis and clinical outcomes assessment}

Treatment failure was defined as conversion to TKA or removal of implant. Unicompartmental knee replacement for the opposite compartment was not considered as treatment failure as per a recent study [1]. Survival analysis was performed using the Kaplan-Meier method, with treatment failure as the endpoint. The modified activity Cincinnati Knee Rating Scale [19], the Western Ontario and McMaster Universities Osteoarthritis Index (WOMAC) [2], the Visual Analog Scale (VAS), and Short Form-36 (SF-36) [3] were used to evaluate clinical outcomes. Patients also self-reported knee function and satisfaction. The original Cincinnati Knee Rating Scale is based on a 0-100 continuous scale [21], whereas the modified Cincinnati Knee Rating Scale is based on a 1-10 categorized scale, with a two-point change being considered clinically meaningful (Fig. 2) [4]. Scores were collected pre-operatively and at yearly intervals post-operatively during an office visit or by mailed questionnaire. Additional analyses were performed on the two major cohorts, "virgin" knees or multiply operated knees to see if there was a difference in baseline characteristic and functional outcomes. 

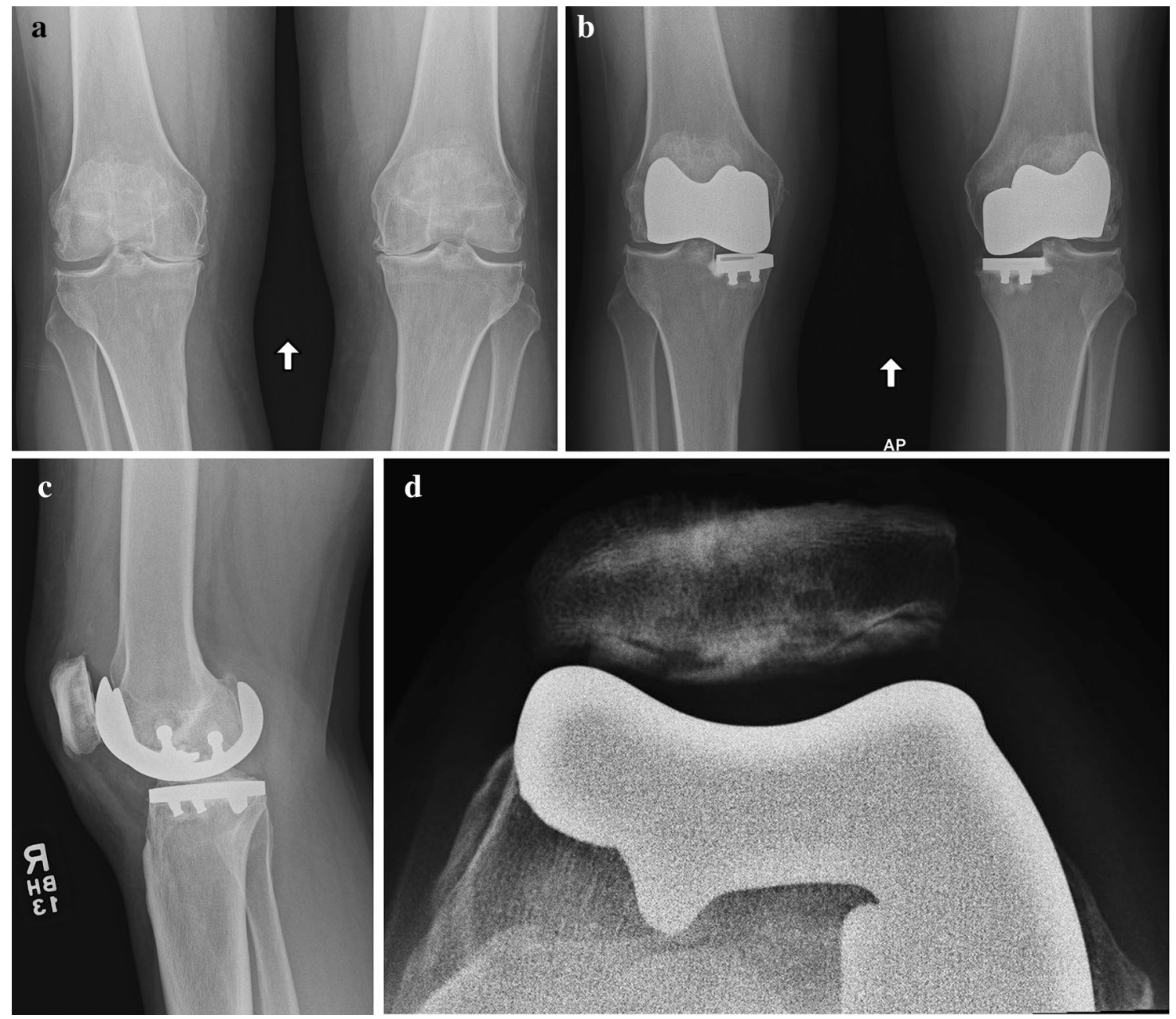

Fig. 1 Bicompartmental knee arthroplasty (BKA). Pre-operative bilateral anteroposterior radiograph of a 44-year-old woman with disabling anterior and medial joint pain (a). Post-operative bilat- eral anteroposterior (b), lateral (c), and skyline (d) radiographs of a patient after bilateral BKA combined medial and patellofemoral replacement

\begin{tabular}{|l|l|l|}
\hline & $\begin{array}{l}\square^{1} \\
\text { POOR }\end{array}$ & $\square^{3} \quad \square^{4} \quad \square^{5} \quad \square^{6} \quad \square^{7} \quad \square^{8} \quad \square^{9} \quad \square^{10}$ \\
\hline Poor & $(1-2)$ & I have significant limitations that affect activities of daily living. \\
\hline Fair & $(3-4)$ & $\begin{array}{l}\text { I have moderate limitations that affect activities of daily living. No } \\
\text { sports possible. }\end{array}$ \\
\hline Good & $(5-6)$ & $\begin{array}{l}\text { I have some limitations with sports but I can participate; I } \\
\text { compensate. }\end{array}$ \\
\hline Very Good $\quad(7-8)$ & I have only a few limitations with sports. \\
\hline Excellent & $(9-10)$ & I am able to do whatever I wish (any sport) with no problem. \\
\hline
\end{tabular}

Fig. 2 Modified Cincinnati Knee Rating scales 


\section{Post-operative radiographic evaluation}

For analyzing the undercoverage or overhang of tibia tray and femoral component, post-operative AP radiographs were reviewed. Undercoverage was defined as the distance between the implant and the edge of the tibial plateau/femoral condyle. Overhang was defined as the distance between the edge of the tibia/femoral condyle and the edge of the tibial tray/femoral component. Undercoverage or overhang of the femoral component or tibial tray at the joint line was measured on a digital picture archiving and communication system (PACS) on calibrated DICOM radiographs.

This study was approved by the Institutional Review Board of Cartilage Repair Center, Brigham and Women's Hospital, Harvard Medical School, Boston, Massachusetts (2007P000470), and written consent was obtained from all patients.

\section{Statistical analysis}

All statistical analyses were performed with Stata (version 13; Statacorp LP, College Station, TX). Kaplan-Meier curves were used for the survival analyses. The Wilcoxon signed-rank test was used to compare differences in functional scores (obtained from the modified Cincinnati, VAS, WOMAC, and SF-36) between the two time points (preoperatively and at the final follow-up). Mann-Whitney $U$ tests were used to compare the improvement in scores between different groups. The level of significance was set a priori at $p<0.05$.

No sample size calculation was performed before conducting this study, because all patients who met the inclusion criteria were included. A post hoc power analysis was conducted using $\mathrm{G}^{*}$ Power, version 3.1. A post hoc power analysis revealed a power of greater than $80 \%$ regarding all the functional scores except for WOMAC stiffness and WOMAC function after BKA-MP, which indicated that sample size was adequate to provide statistical significance to most of the observed differences.

Radiological measurements were performed twice by the same observer. The intra-observer reliability of these measurements was evaluated by an intraclass correlation coefficient. The intra-observer reliability was 0.975 .

\section{Results}

\section{Survival analysis}

Among all 59 knees, 3 knees (5\%) were considered to be failure ( 2 in BKA-MP and 1 in BKA-LP) at an average of
3.1 years post-operatively. Overall, survival rates were $98 \%$ and $92 \%$ at 2 and 5 years, respectively (Fig. 3).

\section{Functional outcomes}

Of 52 patients ( 56 knees) who did not require conversion to TKA, all patient-reported functional scores were clinically meaningfully and significantly improved post-operatively. Of 39 knees successfully treated with BKA-MP, all functional scores except WOMAC stiffness and WOMAC function significantly improved post-operatively. Of 17 knees successfully treated with BKA-LP, all functional scores significantly improved post-operatively (Table 3).

\section{Patient satisfaction survey}

Of patients with retained implants, $91 \%$ of the patients rated their knees as good or excellent, and answered that they were satisfied their knee surgery at the latest follow-up (Table 4).

\section{Radiographic evaluation}

In all cases, radiographic evaluation demonstrated an ideal and perfect fit of the femoral components. For the tibial components, $95 \%$ of the components fit within less than $2 \mathrm{~mm}$ of undercoverage or overhang (including $66 \%$ being fitted perfectly). No tibial components mismatched greater than $3 \mathrm{~mm}$ (Table 5).

\section{Subanalysis: "virgin" knees vs. "multiply operated" knees}

Of 41 patients who underwent BKA-MP, 13 patients constituted the cohort of the "virgin" knees and 28 patients constituted the cohort of the "multiply operated" knees.

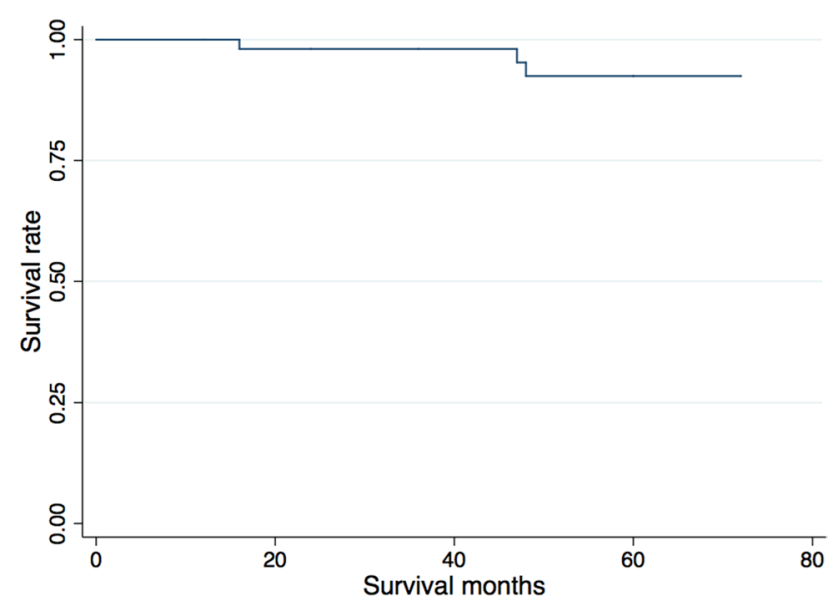

Fig. 3 Kaplan-Meier survival curve. Overall ( $n=59$ knees), survival rates were $98 \%$ and $92 \%$ at 2 and 5 years, respectively 
Table 3 Pre-operative and final follow-up clinical outcomes in successfully treated knees with BKA-MP $(n=39$ knees $)$ and BKA-LP ( $n=17$ knees)
Table 4 Satisfaction with the procedure at final follow-up (in the 56 successful knees)

\begin{tabular}{|c|c|c|c|c|c|c|}
\hline \multirow[t]{2}{*}{ Rating system } & \multicolumn{3}{|c|}{ BKA-MP $(n=39)$} & \multicolumn{3}{|c|}{ BKA-LP $(n=17)$} \\
\hline & Pre-operative & Final follow-up & $P$ value & Pre-operative & Final follow-up & $P$ value \\
\hline Modified Cincinnati & $1.9 \pm 1.0$ & $6.1 \pm 1.9$ & $<0.001$ & $1.9 \pm 0.8$ & $6.4 \pm 2.0$ & $<0.001$ \\
\hline VAS & $6.3 \pm 2.2$ & $2.1 \pm 1.4$ & $<0.001$ & $5.5 \pm 1.7$ & $1.5 \pm 1.3$ & $<0.001$ \\
\hline WOMAC total & $36.3 \pm 16.9$ & $28.2 \pm 15.9$ & 0.0092 & $36.6 \pm 16.5$ & $19.9 \pm 13.7$ & 0.0018 \\
\hline WOMAC_pain & $8.3 \pm 3.8$ & $5.4 \pm 3.7$ & $<0.001$ & $8.1 \pm 3.6$ & $3.6 \pm 2.8$ & $<0.001$ \\
\hline WOMAC_-stiffness & $3.7 \pm 1.7$ & $3 \pm 1.4$ & ns & $3.5 \pm 1.9$ & $2.2 \pm 1.6$ & 0.0129 \\
\hline WOMAC-function & $24.3 \pm 12.5$ & $20 \pm 11.6$ & ns & $25.1 \pm 11.6$ & $14.1 \pm 9.7$ & 0.0036 \\
\hline SF-36-PCS & $38.6 \pm 7.6$ & $47.8 \pm 8.4$ & $<0.001$ & $38.5 \pm 6.6$ & $47.2 \pm 10.4$ & 0.0245 \\
\hline SF-36-MCS & $40.5 \pm 6.5$ & $52.6 \pm 9.9$ & $<0.001$ & $40.5 \pm 7.2$ & $51.7 \pm 10.6$ & 0.0031 \\
\hline
\end{tabular}

$B K A-M P$ bicompartmental knee arthroplasty combined medial and patellofemoral replacement, $B K A-L P$ bicompartmental knee arthroplasty combined lateral with patellofemoral replacement, VAS Visual Analog Scale, WOMAC Western Ontario and McMaster Universities Osteoarthritis Index SF-36, Short Form-36, $M C S$ mental component score, $P C S$ physical component score, $n s$ not significant

\begin{tabular}{|c|c|c|c|}
\hline Question & $\begin{array}{l}\text { BKA-MP } \\
(n=39 \\
\text { knees })\end{array}$ & $\begin{array}{l}\text { BKA-LP } \\
(n=17 \\
\text { knees })\end{array}$ & $\begin{array}{l}\text { Overall } \\
(n=56 \\
\text { knees })\end{array}$ \\
\hline \multicolumn{4}{|c|}{ What is your overall satisfaction level with the joint surgery? } \\
\hline Satisfied & $35(90 \%)$ & $16(94 \%)$ & $51(91 \%)$ \\
\hline Neutral & $2(5 \%)$ & $0(0 \%)$ & $2(4 \%)$ \\
\hline Dissatisfied & $2(5 \%)$ & $1(6 \%)$ & $3(5 \%)$ \\
\hline \multicolumn{4}{|c|}{ How would you rate the results of your joint surgery? } \\
\hline Good/excellent & $36(92 \%)$ & $15(88 \%)$ & $51(91 \%)$ \\
\hline Fair & $2(5 \%)$ & $2(12 \%)$ & $4(7 \%)$ \\
\hline Poor & $1(3 \%)$ & 0 & $1(2 \%)$ \\
\hline \multicolumn{4}{|c|}{$\begin{array}{l}\text { If you could go back in time and make the decision again, } \\
\text { would you choose to undergo your joint surgery? }\end{array}$} \\
\hline Yes & $36(92 \%)$ & $16(94 \%)$ & $52(96 \%)$ \\
\hline Uncertain & $2(5 \%)$ & 0 & $2(2 \%)$ \\
\hline No & $1(3 \%)$ & $1(6 \%)$ & $2(2 \%)$ \\
\hline
\end{tabular}

$B K A-M P$ bicompartmental knee arthroplasty combined medial and patellofemoral replacement, $B K A-L P$ bicompartmental knee arthroplasty combined lateral with patellofemoral replacement
Table 5 Post-operative radiographic evaluation

\begin{tabular}{llc}
\hline & Femoral component & $\begin{array}{l}\text { Tibial } \\
\text { component } \\
(\%)\end{array}$ \\
\hline 2-3 mm undercoverage & 0 & $2(3.4)$ \\
1-2 mm undercoverage & 0 & $4(6.8)$ \\
0-1 mm undercoverage & 0 & $3(5.1)$ \\
Perfect matching & $59(100 \%)$ & $39(66.1)$ \\
0-1 mm overhang & 0 & $6(10.2)$ \\
1-2 mm overhang & 0 & $4(6.8)$ \\
2-3 mm overhang & 0 & $1(1.7)$ \\
\hline
\end{tabular}

Patient age at the time of surgery was significantly older in the "Virgin" than "multiply operated" knees $(P=0.0247)$. The other variables showed no significant difference between the two cohorts. Of 18 patients who underwent BKA-LP, 4 patients constituted the cohorts of "virgin" knees and 14 patients constituted the cohorts of "multiply operated" knees. There was no significant difference in baseline characteristics between the two cohorts (Table 6).

Pre- and post-operative functional scores were compared for the two cohorts (Tables 7, 8). Of patients who underwent BKA-MP, pre-operative scores of VAS, WOMAC total, WOMAC pain, and WOMAC function were significantly lower in the "virgin" knees than the "multiply operated" knees. There was no significant difference in all post-operative scores between those cohorts. 
Table 6 Patient demographics (virgin vs. multiply operated knee)
Virgin knee $(n=17$ knees $) \quad$ Multiply operated $\quad P$ value knee $(n=42$ knees $)$

\begin{tabular}{llll}
\hline Variables & & & \\
BKA-MP & $n=13$ & $n=28$ & \\
Age at surgery (years), Mean \pm SD (range) & $55.2 \pm 5.8(45-65)$ & $50 \pm 6.8(41-63)$ & 0.0247 \\
Gender, male/female, $n$ & $6 / 7$ & $10 / 18$ & $\mathrm{~ns}$ \\
Right/left knee, $n$ & $7 / 6$ & $15 / 13$ & $\mathrm{~ns}$ \\
Body mass index $\left(\mathrm{kg} / \mathrm{m}^{2}\right)$, mean $\pm \mathrm{SD}($ range) & $28 \pm 3.1(22.2-33)$ & $29 \pm 4.2(20.1-39.3)$ & $\mathrm{ns}$ \\
Previous surgeries, mean $\pm \mathrm{SD}($ range) & - & $3.2 \pm 2.8(1-13)$ & - \\
BKA-LP & $n=4$ & $n=14$ & \\
Age at surgery (years), mean $\pm \mathrm{SD}$ (range) & $53 \pm 3.2(50-57)$ & $47.4 \pm 6.6(37-60)$ & $\mathrm{ns}$ \\
Gender, male/female, $n$ & $0 / 4$ & $8 / 6$ & $\mathrm{~ns}$ \\
Right/left knee, $n$ & $3 / 1$ & $7 / 7$ & $\mathrm{~ns}$ \\
Body mass index $\left(\mathrm{kg} / \mathrm{m}^{2}\right)$, mean \pm SD (range) & $20.5 \pm 1.7(19.2-22.9)$ & $29 \pm 6.0(20.7-39.3)$ & 0.0137 \\
Previous surgeries, mean $\pm \mathrm{SD}($ range) & - & $3.6 \pm 2.9(1-10)$ & - \\
\hline
\end{tabular}

$B K A-M P$ bicompartmental knee arthroplasty combined medial and patellofemoral replacement, $B K A-L P$ bicompartmental knee arthroplasty combined lateral with patellofemoral replacement, $n s$ not significant
Of those who underwent BKA-LP, there was no significant difference in all pre- and post-operative scores between those cohorts.

\section{Subsequent surgical procedures (SSPs)}

Other than these three failed knees, 17 knees (30\%) required SSPs (Table 9). Of the 16 "virgin" knees who did not have the previous surgery before the index surgery, 3 knees $(3 / 16,18.8 \%)$ required SSPs, whereas, of those with prior multiple surgeries, 14 knees $(14 / 40,35 \%)$ required SSPs. One patient had distal femoral extension osteotomy with an allograft medial collateral ligament reconstruction (MCLR) in 1 at 2 years to obtain hyperextension of $15^{\circ}$, which he had before BKA and neutral alignment to resolve his inward thrust after BKA. This patient had polio in the Middle East and had Grade 2/5 extensor mechanism weakness requiring hyperextension to lock his knee and walk without assistive device. Other complications including deep venous thrombosis or wound infection were not observed. No patient required any blood transfusion or blood products.

\section{Discussion}

The most important finding of the present study was that CIM-BKA provides very high level of satisfaction with a significant improvement in pain, function, and mental health in young arthritic patients over a short- to mid-term followup by allowing precise fit of femoral components with $66 \%$ of tibial components being precisely fit and $95 \%$ being less than $2 \mathrm{~mm}$ of undercoverage or overhang.

Although substantial improvement in clinical outcomes is reported after TKA in young patients, increased risks of failure rates, less improvement, and dissatisfaction after TKA in those young and early OA patients have been shown [10, 26]. Moreover, a recent systematic review showed that, in patients under 65 years old, unicompartmental knee arthroplasty (UKA) provided higher functional outcomes but higher revision rates than TKA [14]. In young and early OA patients who are difficult to treat, a high level of satisfaction observed in this study, $91 \%$, could be due to restoration of anatomical ' $\mathrm{J}$ ' curves leading to more normal kinematics [30] and preservation of ACL and PCL, leading to a more normal proprioception provided by a customized implant based on each patient's individual anatomy. In addition, the modified activity Cincinnati score showed a mean of 6.1 post-operatively, which indicated that patients were able to participate in sports activities. This achievement might contribute to high level of satisfaction, as well.

The subanalysis revealed that some of the pre-operative functional scores were significantly lower in the multiply operated knees than the "Virgin" knees. Nevertheless, the post-operative functional scores in both cohorts were comparable. Several studies reported the negative effects of the previous surgeries on the outcomes after TKR [10, 24, 25]. However, our results indicate that the individualized BKA may also be encouraging for those who had multiple previous surgeries unlike other prior studies looking at TKA.

Relatively high rate of SSPs was observed, which might be due to the baseline nature of young multiply operated knees before the index surgery. In fact, the rate of SSPs in the patients who did not have the previous surgeries was 
Table 7 Post-operative functional scores in BKA-MP (virgin vs. multiply)

\begin{tabular}{|c|c|c|c|}
\hline BKA-MP & Virgin knee $(n=12)$ & $\begin{array}{l}\text { Multiply } \\
\text { operated knee } \\
(n=27)\end{array}$ & $P$ value \\
\hline \multicolumn{4}{|c|}{ Modified Cincinnati } \\
\hline Pre & $2.1 \pm 0.8$ & $1.9 \pm 1.1$ & $\mathrm{~ns}$ \\
\hline Post & $6.3 \pm 2.2$ & $6.0 \pm 1.8$ & ns \\
\hline \multicolumn{4}{|l|}{ VAS } \\
\hline Pre & $5.3 \pm 2.4$ & $6.9 \pm 1.9$ & 0.0377 \\
\hline Post & $1.4 \pm 0.8$ & $2.3 \pm 1.6$ & ns \\
\hline \multicolumn{4}{|c|}{ WOMAC total } \\
\hline Pre & $26.9 \pm 10.5$ & $40.5 \pm 17.7$ & 0.0101 \\
\hline Post & $26 \pm 12.0$ & $29.1 \pm 17.2$ & ns \\
\hline \multicolumn{4}{|c|}{ WOMAC pain } \\
\hline Pre & $5.9 \pm 2.4$ & $9.4 \pm 3.8$ & 0.0027 \\
\hline Post & $5.2 \pm 2.1$ & $5.5 \pm 3.8$ & $\mathrm{~ns}$ \\
\hline \multicolumn{4}{|c|}{ WOMAC stiffness } \\
\hline Pre & $3.3 \pm 0.9$ & $3.9 \pm 2.0$ & $\mathrm{~ns}$ \\
\hline Post & $2.9 \pm 0.9$ & $3.0 \pm 1.6$ & ns \\
\hline \multicolumn{4}{|c|}{ WOMAC function } \\
\hline Pre & $17.7 \pm 8.2$ & $27.2 \pm 13.1$ & 0.0280 \\
\hline Post & $17.9 \pm 9.3$ & $20.5 \pm 12.1$ & $\mathrm{~ns}$ \\
\hline \multicolumn{4}{|l|}{ SF-36 PCS } \\
\hline Pre & $38.7 \pm 6.3$ & $38.6 \pm 8.2$ & ns \\
\hline Post & $49.3 \pm 8.4$ & $47.2 \pm 8.4$ & $\mathrm{~ns}$ \\
\hline \multicolumn{4}{|c|}{ SF-36 MCS } \\
\hline Pre & $41.8 \pm 5.3$ & $39.9 \pm 7.0$ & $\mathrm{~ns}$ \\
\hline Post & $55.3 \pm 7.2$ & $51.4 \pm 10.7$ & ns \\
\hline
\end{tabular}

$B K A-M P$ bicompartmental knee arthroplasty combined medial and patellofemoral replacement, VAS Visual Analog Scale, WOMAC Western Ontario and McMaster Universities Osteoarthritis Index SF-36, Short Form-36, MCS mental component score, PCS physical component score, $n s$ not significant

lower (18.8\%) compared to those who had multiple prior surgeries (35\%). The previous surgeries in our study primarily included arthroscopic procedures and HTO. Similar observation was found in a previous study that showed significantly higher post-operative complications (30\%) in patients who had the previous arthroscopic debridement than those who did not (10\%) [24].

Three patients were considered as a treatment failure and converted to TKA. Of those, no patients required stems, wedges, or PS components, which we believe support the philosophy of bone preservation and cruciate preservation. Similar failure rates were reported in patients after UKA [9, 28]. The first patient failed due to loosening of a tibial tray at 4 years, which is a common cause for revision surgery after UKA [1]. The second patient failed due to progression of the opposite compartment, also a common problem with UKA [1]. Because the BKA implant was well fixed, the treatment
Table 8 Post-operative functional scores in BKA-LP (virgin vs. multiply)

\begin{tabular}{|c|c|c|c|}
\hline BKA-LP & Virgin knee $(n=4)$ & $\begin{array}{l}\text { Multiply } \\
\text { operated knee } \\
(n=13)\end{array}$ & $P$ value \\
\hline \multicolumn{4}{|c|}{ Modified Cincinnati } \\
\hline Pre & $2 \pm 0$ & $1.8 \pm 0.9$ & ns \\
\hline Post & $7.8 \pm 1.7$ & $6 \pm 1.9$ & ns \\
\hline \multicolumn{4}{|l|}{ VAS } \\
\hline Pre & $5.8 \pm 1.9$ & $5.5 \pm 1.7$ & ns \\
\hline Post & $0.8 \pm 1.0$ & $1.7 \pm 1.4$ & $\mathrm{~ns}$ \\
\hline \multicolumn{4}{|c|}{ WOMAC total } \\
\hline Pre & $34.5 \pm 3.4$ & $37.3 \pm 18.9$ & ns \\
\hline Post & $23.5 \pm 9.8$ & $18.8 \pm 14.8$ & ns \\
\hline \multicolumn{4}{|c|}{ WOMAC pain } \\
\hline Pre & $7.5 \pm 1.3$ & $8.3 \pm 4.1$ & ns \\
\hline Post & $4 \pm 2.7$ & $3.5 \pm 2.9$ & ns \\
\hline \multicolumn{4}{|c|}{ WOMAC stiffness } \\
\hline Pre & $3 \pm 0.82$ & $3.6 \pm 2.1$ & ns \\
\hline Post & $2.3 \pm 1.7$ & $2.2 \pm 1.6$ & ns \\
\hline \multicolumn{4}{|c|}{ WOMAC function } \\
\hline Pre & $24 \pm 3.4$ & $25.4 \pm 13.2$ & ns \\
\hline Post & $17.5 \pm 5.7$ & $13.1 \pm 10.6$ & $\mathrm{~ns}$ \\
\hline \multicolumn{4}{|c|}{ SF-36 PCS } \\
\hline Pre & $33.7 \pm 7.8$ & $40.0 \pm 5.7$ & ns \\
\hline Post & $48.1 \pm 13.6$ & $46.9 \pm 9.9$ & $\mathrm{~ns}$ \\
\hline \multicolumn{4}{|c|}{ SF-36 MCS } \\
\hline Pre & $38.5 \pm 6.4$ & $41.2 \pm 7.6$ & ns \\
\hline Post & $56.2 \pm 4.6$ & $50.3 \pm 11.7$ & ns \\
\hline
\end{tabular}

$B K A-L P$ bicompartmental knee arthroplasty combined lateral with patellofemoral replacement, VAS Visual Analog Scale, WOMAC Western Ontario and McMaster Universities Osteoarthritis Index SF-36, Short Form-36, MCS mental component score, PCS physical component score, $n s$ not significant

Table 9 Subsequent surgical procedures

\begin{tabular}{lcl}
\hline Procedures & Knees, $n$ & Timing post-operatively, years \\
\hline A/S synovectomy & 13 & $1.4 \pm 1.1$ (range 0.2-3.6) \\
A/S meniscectomy & 2 & 0.8 and 3.2 \\
UKR & 2 & 0.9 and 1.9 \\
Osteotomy with MCLR & 1 & 2
\end{tabular}

$A / S$ arthroscopic, UKR unicompartmental knee replacement, MCLR medial collateral ligament reconstruction

options included unicompartmental arthroplasty with retaining BKA implant rather than TKA. However, the patient underwent TKA due to her preference. The third patient, an obese, BMI $41 \mathrm{~kg} / \mathrm{m}^{2}$, diabetic male developed a late infection after an initial excellent post-operative course and outcome, requiring a staged explant with antibiotic cement 
spacer and a primary CR TKA. Two patients who required unicompartmental arthroplasty on the opposite compartment after the CIM-BKA were not considered as a treatment failure as the BKA was well fixed and a lateral UKA dealt with the progression of OA-preserving bone stock and both cruciates. Clarifying a risk factor for the progression of $\mathrm{OA}$ on the remaining compartment will be necessary to avoid multiple replacement surgery. Most recently, a multi-center study showed the implant survivor rate after Oxford UKA was $90 \%$ at 10 years using the same approach, not including unicompartmantal arthroplasty on the opposite compartment as a failure [1]. The survival rates in this study $(98 \%$ and $92 \%$ at 2 and 5 years, respectively) were comparable, although a long-term follow-up is still necessary to confirm the durability of this unique implant.

Although BKA theoretically can provide better functional outcomes due to the preservation of ligaments, proprioception, and distribution of joint loading, controversy still exists regarding the superiority of BKA over TKA. Our study showed that patient-specific BKA is promising over a shortto mid-term follow-up without any concerns about the gap between transitional edge of the trochlear component and the proximal edge of the native femoral articular surface as it is monolithic and designed to "dive" $5 \mathrm{~mm}$ deep to the subchondral bone based on the planning CT scan. In addition, it can offer an option for lateral OA combined with PF OA which only could have been manageable with an unlinked implant using implants manufactured basically for medial tibiofemoral OA instead of for lateral tibiofemoral OA.

A previously reported "off-the-shelf" monolithic implant required surgeons to implant the definitive implantation on the coronal plane to best resurface the medial and PF compartments [8, 22]. Conversely, a patient-customized implant can cover each patient-specific resurfacing precisely as demonstrated by our X-ray analyses fit of the femoral implant in all operated knees and precise fit of the tibial implant in $66 \%$ of all patients. Although $34 \%$ of patients did not show a precise fit of the tibial implant, the findings that $95 \%$ of all patients showed less than $2 \mathrm{~mm}$ of undercoverage or overhang are encouraging. The amount of osteophytes that are needed to be removed during the surgery depends on the surgeon, although the I-view protocol suggests the estimated osteophytes that should be removed. Thus, it might be difficult to determine the precise volume of osteophytes that should be removed during the surgery, which might have resulted in not perfectly precise fit in some cases. The previous studies have shown that an overhang of $\geq 3 \mathrm{~mm}$ in femoral and tibial component coverage was associated with an increased risk for knee pain after TKA [15] and UKA [5]. In the present study, no patients had undercoverage or overhang greater than $3 \mathrm{~mm}$ on a digital radiograph. Another human cadaveric study demonstrated overhang of the tibial tray greater than $2 \mathrm{~mm}$ can load and impinge on the medial collateral ligament and possibly cause knee pain [12]. Thus, a precise fit of the implant may be crucial to preventing softtissue irritation with pain and CIM- BKA is encouraging.

There were several limitations in this study. First, this is a case series with no control groups and not a randomized clinical trial against TKA or BKA with linked uni and PF replacements. However, thankfully, it is very difficult to find patients who have had multiple previous surgeries before prosthetic arthroplasty. Second, this study is short- to midterm follow-up study. Thus, longer follow-up study will be needed to confirm the durability of this implant and whether patient functional outcomes and high level of satisfaction are maintained. Third, we measured overhang and undercoverage using digital radiographs which are not as accurate as measurement based on CT. However, prior studies have used post-operative radiographs to evaluate the fit of the implant $[6,7]$. Fourth, we did not evaluate pre- and post-operative range of motion of the knee. This evaluation will be necessary in the long-term follow-up study. Finally, we did not include patients who had UKR on the opposite compartment. However, our definition for failure was consistent with the previous study [1].

\section{Conclusion}

This customized resurfacing implant provided a precise fit of femoral components and a precise fit of $66 \%$ of tibial components with $95 \%$ of tibias being less than $2 \mathrm{~mm}$ of undercoverage or overhang. This novel resurfacing implant provided a high level of patient satisfaction with significant improvement in pain, function, and mental health over the short- to mid-term follow-up for the treatment of bicompartmental OA in young arthritic patients, whose average age was 51 in this study. Although there was a relatively high rate of SSPs observed particularly in patients with multiply operated knees, CIM-BKA allows the surgeon to achieve individualized optimal implantation and may be a satisfactory option for bicompartmental OA. A longer term followup is necessary to determine its role in the knee arthroplasty armamentarium.

Authors' contributions TO analyzed data and drafted the manuscript. $\mathrm{KL}, \mathrm{GM}$, and TB participated in the design of the study. TM conceived the study, participated in its design and coordination, and helped to draft the manuscript. All authors read and approved the final manuscript.

Funding Takahiro Ogura has received funding from the Cartilage Research Foundation, Indianapolis, Indiana, USA. Gergo Merkely has received funding from the Rosztoczy Foundation. 


\section{Compliance with ethical standards}

Conflict of interest Tom Minas is a consultant to Vericel, SAB member (stocks and royalties) of Conformis, and has book royalties for sales (Elsevier). The other authors have nothing to declare.

Ethic approval Ethical approval was obtained from institutional review board.

Open Access This article is distributed under the terms of the Creative Commons Attribution 4.0 International License (http://creativeco mmons.org/licenses/by/4.0/), which permits unrestricted use, distribution, and reproduction in any medium, provided you give appropriate credit to the original author(s) and the source, provide a link to the Creative Commons license, and indicate if changes were made.

\section{References}

1. Alnachoukati OK, Barrington JW, Berend KR, Kolczun MC, Emerson RH, Lombardi AV Jr et al (2018) Eight hundred twentyfive medial mobile-bearing unicompartmental knee arthroplaties: the first 10-year us multi-center survival analysis. J Arthroplasty 33:677-683

2. Bellamy N, Buchanan WW, Goldsmith CH, Campbell J, Stitt LW (1988) Validation study of WOMAC: a health status instrument for measuring clinically important patient relevant outcomes to antirheumatic drug therapy in patients with osteoarthritis of the hip or knee. J Rheumatol 15:1833-1840

3. Brazier JE, Harper R, Jones NM, O'Cathain A, Thomas KJ, Usherwood T et al (1992) Validating the SF-36 health survey questionnaire: new outcome measure for primary care. BMJ 305:160-164

4. Browne JE, Anderson AF, Arciero R, Mandelbaum B, Moseley JB Jr, Micheli LJ et al (2005) Clinical outcome of autologous chondrocyte implantation at 5 years in US subjects. Clin Orthop Relat Res 436:237-245

5. Chau R, Gulati A, Pandit H, Beard DJ, Price AJ, Dodd CA et al (2009) Tibial component overhang following unicompartmental knee replacement-does it matter? Knee 16:310-313

6. Clarke HD, Hentz JG (2008) Restoration of femoral anatomy in TKA with unisex and gender-specific components. Clin Orthop Relat Res 466:2711-2716

7. Demange MK, Von Keudell A, Probst C, Yoshioka H, Gomoll AH (2015) Patient-specific implants for lateral unicompartmental knee arthroplasty. Int Orthop 39:1519-1526

8. Dudhniwala AG, Rath NK, Joshy S, Forster MC, White SP (2016) Early failure with the Journey-Deuce bicompartmental knee arthroplasty. Eur J Orthop Surg Traumatol 26:517-521

9. Ernstbrunner L, Imam MA, Andronic O, Perz T, Wieser K, Fucentese SF (2017) Lateral unicompartmental knee replacement: a systematic review of reasons for failure. Int Orthop. https://doi. org/10.1007/s00264-017-3662-4

10. Frank RM, Della Valle CJ, Plummer DR, Chalmers PN, Cole BJ (2017) Does prior cartilage restoration impact outcomes following knee arthroplasty? Orthop Clin North Am 48:265-273

11. Gokeler A, Benjaminse A, Hewett TE, Lephart SM, Engebretsen L, Ageberg E et al (2012) Proprioceptive deficits after ACL injury: are they clinically relevant? Br J Sports Med 46:180-192

12. Gudena R, Pilambaraei MA, Werle J, Shrive NG, Frank CB (2013) A safe overhang limit for unicompartmental knee arthroplasties based on medial collateral ligament strains: an in vitro study. J Arthroplasty 28:227-233

13. Kellgren JH, Lawrence JS (1957) Radiological assessment of rheumatoid arthritis. Ann Rheum Dis 16:485-493
14. Kleeblad LJ, van der List JP, Zuiderbaan HA, Pearle AD (2018) Larger range of motion and increased return to activity, but higher revision rates following unicompartmental versus total knee arthroplasty in patients under 65: a systematic review. Knee Surg Sports Traumatol Arthrosc 26:1811-1822

15. Mahoney OM, Kinsey T (2010) Overhang of the femoral component in total knee arthroplasty: risk factors and clinical consequences. J Bone Joint Surg Am 92:1115-1121

16. McCurry SM, Von Korff M, Vitiello MV, Saunders K, Balderson BH, Moore AL et al (2011) Frequency of comorbid insomnia, pain, and depression in older adults with osteoarthritis: predictors of enrollment in a randomized treatment trial. J Psychosom Res 71:296-299

17. McDonough CM, Jette AM (2010) The contribution of osteoarthritis to functional limitations and disability. Clin Geriatr Med 26:387-399

18. Meehan JP, Danielsen B, Kim SH, Jamali AA, White RH (2014) Younger age is associated with a higher risk of early periprosthetic joint infection and aseptic mechanical failure after total knee arthroplasty. J Bone Joint Surg Am 96:529-535

19. Micheli LJ, Browne JE, Erggelet C, Fu F, Mandelbaum B, Moseley JB et al (2001) Autologous chondrocyte implantation of the knee: multicenter experience and minimum 3-year follow-up. Clin J Sport Med 11:223-228

20. Minas T (2011) A primer in cartilage repair and joint preservation of the knee: expert consult. Elsevier Health Sciences, New York

21. Noyes FR, Barber SD, Mooar LA (1989) A rationale for assessing sports activity levels and limitations in knee disorders. Clin Orthop Relat Res 246:238-249

22. Palumbo BT, Henderson ER, Edwards PK, Burris RB, Gutierrez S, Raterman SJ (2011) Initial experience of the Journey-Deuce bicompartmental knee prosthesis: a review of 36 cases. J Arthroplasty $26: 40-45$

23. Parratte S, Ollivier M, Opsomer G, Lunebourg A, Argenson JN, Thienpont E (2015) Is knee function better with contemporary modular bicompartmental arthroplasty compared to total knee arthroplasty? Short-term outcomes of a prospective matched study including 68 cases. Orthop Traumatol Surg Res 101:547-552

24. Piedade SR, Pinaroli A, Servien E, Neyret P (2009) Is previous knee arthroscopy related to worse results in primary total knee arthroplasty? Knee Surg Sports Traumatol Arthrosc 17:328-333

25. Piedade SR, Pinaroli A, Servien E, Neyret P (2013) TKA outcomes after prior bone and soft tissue knee surgery. Knee Surg Sports Traumatol Arthrosc 21:2737-2743

26. Scott CE, Oliver WM, MacDonald D, Wade FA, Moran M, Breusch SJ (2016) Predicting dissatisfaction following total knee arthroplasty in patients under 55 years of age. Bone Joint $\mathbf{J}$ 98-b:1625-1634

27. Steinert AF, Beckmann J, Holzapfel BM, Rudert M, Arnholdt J (2017) Bicompartmental individualized knee replacement: use of patient-specific implants and instruments (iDuo). Oper Orthop Traumatol 29:51-58

28. van der List JP, Zuiderbaan HA, Pearle AD (2016) Why do medial unicompartmental knee arthroplasties fail today? J Arthroplasty 31:1016-1021

29. Von Keudell A, Sodha S, Collins J, Minas T, Fitz W, Gomoll AH (2014) Patient satisfaction after primary total and unicompartmental knee arthroplasty: an age-dependent analysis. Knee $21: 180-184$

30. Wunschel M, Lo J, Dilger T, Wulker N, Muller O (2011) Influence of bi- and tri-compartmental knee arthroplasty on the kinematics of the knee joint. BMC Musculoskelet Disord 12:29

31. Yeo NE, Chen JY, Yew A, Chia SL, Lo NN, Yeo SJ (2015) Prospective randomised trial comparing unlinked, modular bicompartmental knee arthroplasty and total knee arthroplasty: a five years follow-up. Knee 22:321-327 